healthcare staff is imperative to drive improvement and increase adherence rates.

\section{Identifying Risk Factors for Re-admission: A Service Evaluation from an Adult Inpatient Mental Health Unit}

Kirsty Ward ${ }^{\star}$ and Suveera Prasad

Rotherham, Doncaster and South Humber NHS Foundation Trust ${ }^{*}$ Corresponding author.

doi: 10.1192/bjo.2021.201

Aims. To identify risk factors for re-admission to an acute inpatient general adult mental health ward. There is need to ensure that mental health services adapt to the increasing demand for inpatient beds Method. We conducted a single centre retrospective analysis of electronic records of 85 discharges from an adult mental health unit from 4th March 2019 - 5th August 2019. We collected information on demographics, admission details, substance use, forensic history, diagnosis as per the International Classification of Diseases 10th Edition (ICD-10), and discharge details and compared two cohorts; those re-admitted within three months of discharge and those who were not. Odds ratio (OR), 95\% confidence intervals $(\mathrm{CI})$ and $\mathrm{p}$ values were calculated where possible.

Result. Among seventeen service users who were re-admitted within the three month period there were nine women and eight men. There was no difference in ethnicity, employment or marital status. The mean length of admission for those readmitted was 48.2 days (range 1-140 days) and 47.1 days (range 1-350 days) for those who were not readmitted. Certain features were more prevalent among the readmitted group including forensic history $(58.8 \%$ [10] vs $26.5 \%$ [18], OR 3.97, CI 1.31-11.9, p value 0.007 ), substance misuse history (70.6\% [12] vs 55.9\% [38], OR 1.89, CI 0.60-5.97, p value 0.138$)$, previous contact with mental health services $(100 \%$ [17] vs 76.5\% [52]) and the rate of detention under the Mental Health Act at point of admission (76.5\% [13] vs $66.2 \%$ [45], OR 1.66 , CI $0.49,5.67$, p value 0.209 ).

Among those readmitted, a diagnosis of emotionally unstable personality disorder $(17.6 \%$ [3] vs $10.3 \%$ [7], OR $1.87, \mathrm{CI}$ $0.43,-8.14$, p values 0.203$)$ and substance misuse disorder $(41.2 \%$ [7] vs $17.6 \%$ [12], OR 3.27, CI 1.04-10.31, p value 0.218 ) were more prevalent. They were more likely to use illicit substances whilst they were an inpatient (23.5\% [4] versus $7.6 \%$ [5], OR 3.88, CI $0.92-$ 16.43 , $\mathrm{p}$ value 0.033$)$ and to be involved in police incidents $(35.3 \%$ [6] versus $17.6 \%$ [12], OR 2.55, CI 0.79-8.23, p value 0.059 ).

Conclusion. Our trends demonstrate that people with substance misuse, emotionally unstable personality disorder and forensic history are more likely to be readmitted to an adult mental health inpatient unit. They were more likely to misuse illicit substances and be involved with police during admission.

Evaluation of a novel consultant psychiatric clinic in general practices and its effects on secondary mental health contact and the general practitioners' perspectives

Kamran Mahmood ${ }^{1 \star}$, Daniel Whitney ${ }^{2}$ and Guy Brookes ${ }^{1}$

${ }^{1}$ Leeds and York Partnership Foundation Trust and ${ }^{2}$ Tees Esk and Wear Valleys NHS Foundation Trust

${ }^{\star}$ Corresponding author.

doi: 10.1192/bjo.2021.202
Aims. To assess whether direct access to a 45 minute screen appointment in a Consultant Psychiatric clinic, based in General Practice, affects; the number of contacts patients have with secondary care pre and post being seen; whether the General Practitioner (GP) would have referred to secondary services if the clinic had not been in operation; the GPs' views on how helpful the clinic was in understanding the patients' problems and managing the problems outside of secondary care.

Background. A Consultant Psychiatrist in Leeds offered bespoke 45 minute screening appointment clinics in three sister GP practices, accepting direct referrals from GPs without requiring referrals to the local Community Mental Health Team (CMHT). This model was created to reduce the number of patients moving repeatedly between GP and secondary mental health services as this was leading to patient dissatisfaction and increased GP and CMHT workloads.

Method. We compared the number of mental health contacts (per month), for each of the 57 patients who had been referred to the clinic, in the months pre and post being seen in the clinic. We also asked the involved GPs to complete a brief survey for each patient who had been referred to determine whether, they would otherwise have been referred to the CMHT and whether the clinic has helped with their understanding and management of the patients' problems.

Result. The mean number of contacts with secondary services before being seen in clinic was 3.30 per month compared to 0.44 after being seen. The mean difference of 2.86 is statistically significant on a paired-test with a P Value of 0.0149 (95\% confidence intervals of 0.58 to 5.13). We received 22 survey responses from GPs of patients referred to the clinic including for patients who did not attend. All 22 responses indicated that the patient would have been referred to the CMHT if the clinic had not been available. $95 \%$ were rated as being very helpful or moderately helpful in understanding the patient's problems. $91 \%$ were rated as very helpful or moderately helpful in managing the patients' problems outside secondary care.

Conclusion. Our evaluation has demonstrated that a model of direct access for GPs to a Consultant Psychiatric clinic can reduce referrals and patient contacts with secondary mental health services. GPs have found this model helpful in understanding patients' problems and managing the problems outside of secondary care.

\section{A retrospective case-control service evaluation of} CAARMS scores of patients with autism in York EIP, compared to age matched controls

Daniel Whitney ${ }^{*}$ and Stephen Wright

Tees Esk and Wear Valleys NHS Foundation Trust

${ }^{*}$ Corresponding author.

doi: 10.1192/bjo.2021.203

Aims. Studies show the prevalence of Autism Spectrum Conditions in Early Intervention in Psychosis (EIP) populations is $3.6-3.7 \%$, compared to approximately $1-1.5 \%$ in the general population. The CAARMS (Comprehensive Assessment of At Risk Mental States) is a national tool used by EIP services as a screening tool to bring patients into services and stratify their symptoms to determine what pathway may be most appropriate (First Episode Psychosis pathway (FEP) or At Risk Mental State pathway (ARMS)). As far as we are aware the CAARMS has not been validated in an autistic population. It is our view that several of the questions in the CAARMS may be interpreted differently by people with autism, thus affecting the scores. The aim 
of this evaluation was to identify whether CAARMS scores differ between patients diagnosed with autism and matched controls in York EIP.

Method. From their mental health records, we identified all patients in the service with a diagnosis of autism. We then compared the CAARMS scores, at the time of referral, to those of age matched controls (matched by being in the age range 16-30) without an autism diagnosis, using continuous sampling by date of referral.

Result. 14 patients in the service had a diagnosis of autism and had completed a CAARMS. CAARMS domains are all scored between 0 and 6 (indicating increasing severity or frequency). Compared to the age matched controls, autistic patients had a higher mean difference in their scores for 'Non-Bizarre Ideas' (mean difference of 0.86 for severity and 0.57 for frequency) and 'Disorganised Speech' (mean difference of 0.28 for severity and 0.57 for frequency). These results did not reach statistical significance which was unsurprising given the sample size. The gender split between groups was similar.

Conclusion. Our evaluation suggests a difference in CAARMS scores between patients in our service with a diagnosis of autism and those without. A larger study would be needed to confirm a statistically significant difference and multicentre results would be needed as evidence of generalisability. However, if such a difference were confirmed it might question the validity of CAARMS in autistic patients or suggest that modifications, perhaps in the form of reasonable adjustments to the questions or scoring, were needed to increase the validity in this population. We would suggest that spending extra time checking the patient has understood the intended meaning of the questions in the CAARMS may increase validity, particularly in the 'Non-Bizarre Ideas' domain.

\section{COVID-19 pandemic moral injury in healthcare professionals: a systematic review}

Verity Williams ${ }^{1 \star}$, Rhian Bradley ${ }^{1}$, Rafey Faruqui ${ }^{1}$, Julia Hynes ${ }^{2}$ and Julie Anderson ${ }^{3}$

${ }^{1}$ Kent and Medway NHS and Social Care Partnership Trust, Kent and Medway Medical School; ${ }^{2}$ Kent and Medway Medical School and

${ }^{3}$ University of Kent

${ }^{*}$ Corresponding author.

doi: 10.1192/bjo.2021.204

Aims. Moral injury (MI) refers to psychological distress resulting from witnessing or participating in events which violate an individual's moral code. Originating from military experiences, the phenomenon also has relevance for healthcare professionals dealing with wars, natural disasters and infectious diseases. The deontological basis of medicine prioritises duty to the individual patient over duty to wider society. These values may place healthcare professionals at increased risk of moral injury, particularly in crisis contexts where they may be party to decisions to withdraw or divert care based on resource availability.

We conducted a systematic review of medical literature to understand the extent and clinical and socio-demographic correlates of moral injury during the COVID-19 pandemic.

Method. We conducted a systematic review of reports included in MEDLINE, PsycINFO, BNI, CINAHL, EMBASE, EMCARE and HMIC databases using search terms: "moral injury" AND "covid" OR "coronavirus" OR "pandemic". We also searched Google Scholar and Ovid Database and conducted reference searching. We searched for published quantitative primary research as well as advance online publications and pre-print research. Findings are reported in line with Preferred Reporting Items for Systematic Reviews and MetaAnalyses (PRISMA). Two authors independently assessed the included studies' methodological quality using a seven-item checklist.

Result. Our databases search identified 498 records and other sources identified 4 records. We screened 391 records after removing duplicates. 4 reports met our protocol requirements.

Three papers used cross-sectional designs. One reported longitudinal outcomes of their sample already described in one of the three papers. Only one study used a MI scoring system validated for healthcare professionals. Others used scoring validated in military populations. These papers reported outcomes from 3334 subjects, with a higher proportion of females. The largest study (3006 subjects) reported MI in $41.3 \%$ of their sample. Overall, factors associated with greater MI included: providing direct care to COVID-19 patients; sleep troubles; being unmarried; aged <30 years; female gender; and Buddhist/Taoist faith. Nurses reported a greater severity of MI than physicians. MI significantly correlated with anxiety, depression and burnout. The longitudinal study reported that more stressful and less supportive work environments predicted greater MI at 3 months follow-up.

The average quality assessment score of these studies was 4/7. Conclusion. It is important that we are able to address moral injury awareness training as part of workforce preparedness and burnout prevention during the COVID-19 pandemic and other disaster responses across the globe.

\section{Do Junior Doctors feel confident using Emergency Detention Certificates?}

Sarah Wordie ${ }^{*}$ Alice Troup, Giovana Klefti and Cinzia Giuntoli Royal Edinburgh Hospital

${ }^{*}$ Corresponding author.

doi: 10.1192/bjo.2021.205

Aims. To assess junior doctors understanding of the law surrounding the use of The Mental Health (Care and Treatment) (Scotland) Act 2003 (MHA) with a focus on assessing confidence and knowledge of the use of the emergency detention certificate (EDC). A secondary aim was to use these findings to develop a variety of educational tools to subsequently improve junior doctors understanding in using the MHA.

Method. We created and distributed a comprehensive electronic survey to 152 Foundation Year Two Doctors working in NHS Lothian, Fife and Borders in December 2020. We subsequently interviewed 20 respondents to enquire about additional resources needed to improve knowledge of the MHA. Following on, we completed worked EDC exemplars, created an easily accessible guide with step-by-step instructions on implementing an EDC and devised a checklist pro-forma that can be accessed and inserted into a patient's electronic notes to ensure all necessary steps are completed for the EDC.

Result. 51 doctors (34\%) responded to our survey, of which 10 (19\%) had previously worked in psychiatry and 16 (31\%) had previously completed an EDC. 27 respondents (52\%) reported a lack of self-confidence and knowledge and 26 (51\%) reported a lack of understanding in the legal processes as barriers faced when putting an EDC in place. $23(45 \%)$ respondents were unaware that a Mental Health Officer (MHO) must be contacted to grant an EDC. Respondents who had experience of working in psychiatry reported greater awareness of the MHA. From the focused interviews held, colleagues requested for worked EDC examples, an easily accessible checklist with relevant contact details and an 